\title{
Cor, capacidade de retenção de água e maciez da carne de cordeiro maturada e injetada com cloreto de cálcio
}

[Colour, water holding capacity and tenderness of lamb aged and injected with calcium chloride]

N.M.B.L. Zeola, P.A. Souza, H.B.A. Souza, A.G. Silva Sobrinho, J.C. Barbosa

Faculdade de Ciências Agrárias e Veterinárias

Via de Acesso Prof. Paulo Donato Castellane, s/n

14884-900 - Jaboticabal, SP

\begin{abstract}
RESUMO
Os músculos Biceps femoris, Longissimus e Triceps brachii submetidos aos efeitos do tempo de maturação e da injeção com cloreto de cálcio de cordeiros Morada Nova foram estudados quanto às características de cor, capacidade de retenção de água e maciez. Os cordeiros foram abatidos ao atingirem $25 \mathrm{~kg}$ de peso vivo. Os músculos apresentaram diferenças na cor (luminosidade-L*, teor de vermelho-a* e teor de amarelo-b*) 24 horas após o abate. A maturação tendeu a escurecer a carne. O cloreto de cálcio não modificou a cor das carnes provenientes dos músculos Longissimus e Triceps brachii, entretanto as do Biceps femoris apresentaram-se mais vermelhas quando receberam cloreto de cálcio. Quanto à capacidade de retenção de água, a maturação afetou as carnes do Triceps brachii, mas não influenciou as carnes dos músculos Biceps femoris e Longissimus. O cloreto de cálcio não modificou a capacidade de retenção de água dos músculos avaliados. A maturação influiu sobre a maciez do Biceps femoris e do Longissimus.
\end{abstract}

Palavras-chave: cordeiro, cor, capacidade de retenção de água, maciez, cloreto de cálcio, maturação

\begin{abstract}
Biceps femoris, Longissimus and Triceps brachii muscles from Morada Nova lambs were submitted to ageing and calcium chloride injection. Colour, water holding capacity and tenderness were studied. Lambs were slaughtered weighting $25 \mathrm{~kg}$.. The muscles presented differences in colour (lightness- $L^{*}$, redness- $a^{*}$ and yellowness- $\left.b^{*}\right) 24$ hours after rigor mortis instalation. Ageing intensified redness of the meats. Calcium chloride did not modify the colour of Longissimus and Triceps brachii, however, Biceps femoris became more redness after receiving calcium chloride. In relation to water holding capacity, ageing affected meats from Triceps brachii. However, it did not affect Biceps femoris and Longissimus. The calcium chloride didn't modify the water holding capacity of the muscles. Ageing influenced tenderness of Biceps femoris and Longissimus.
\end{abstract}

Keywords: lamb, ageing, calcium chloride, colour, tenderness, water holding capacity

\section{INTRODUÇÃO}

A preferência pela carne ovina apresenta aspectos comuns, como a busca por carne macia com pouca gordura e muito músculo, comercializada a preços acessíveis (Silva Sobrinho, 2001). Para atender às exigências do mercado consumidor, o setor produtivo precisa conhecer os fatores que interferem nas características físicas e químicas da carne, pois estas determinam sua qualidade e aceitabilidade (Martínez-Cerezo et al., 2005).

Parâmetros de qualidade como a cor e a capacidade de retenção de água são importantes para o consumidor avaliar o produto; a cor apresenta relevância no momento da compra (Sañudo, 2004), já a capacidade de retenção de

Recebido em 8 de agosto de 2006

Aceito em 3 de maio de 2007

E-mail: dzeola@ig.com.br 
água é importante durante o consumo. De acordo com Arima et al. (1997), citados por Peixoto et al. (2002), a carne maturada, mesmo após a equalização da cor, ainda apresenta um gradiente diferenciado quando comparada à carne não maturada.

Sañudo (1992) citou como fatores intrínsecos na variação da capacidade de retenção de água o tipo de músculo, a raça e a idade, e como fatores extrínsecos, a alimentação, o estresse prévio ao abate e as condições após o abate, sendo que o processo de maturação da carne tende a aumentar sua capacidade de retenção de água.

A menor capacidade de retenção de água da carne implica perdas do valor nutritivo pelo exudato liberado, resultando em carne mais seca e com menor maciez. Características de maciez como firmeza e sensações tácteis estão intimamente relacionadas com a capacidade de retenção de água, $\mathrm{pH}$, grau de gordura de cobertura e características do tecido conjuntivo e da fibra muscular (Pardi et al., 2001).

Pesquisa sobre os possíveis efeitos do tempo de maturação e da utilização do cloreto de cálcio na carne ovina, é considerada relevante para a melhoria da qualidade (Osório e Osório, 1999).

Este trabalho teve o objetivo de estudar os efeitos de diferentes períodos de maturação, da injeção de cloreto de cálcio e da interação desses fatores sobre a cor e a capacidade de retenção de água da carne proveniente dos músculos Biceps femoris (patinho), Longissimus e Triceps brachii (coração da paleta) de cordeiros Morada Nova.

\section{MATERIAL E MÉTODOS}

Foram utilizados 24 cordeiros da raça Morada Nova, machos inteiros, com peso vivo médio inicial de $15 \mathrm{~kg}$ (três meses), os quais permaneceram alojados em galpão de confinamento até o abate, em baias coletivas de $12 \mathrm{~m}^{2}$ cada, compostas por seis cordeiros, totalizando quatro baias equipadas com comedouros e bebedouros.

A dieta experimental foi constituída de volumoso - feno de capim coastcross moído -, concentrado protéico, $44,6 \%$ de farelo de soja, concentrado energético, $54,3 \%$ de milho moído, além de $0,61 \%$ de sal iodado e $0,55 \%$ de núcleo mineral ovino (Nutrient..., 1985). A refeição foi fornecida no período matutino, sendo composta pela relação volumoso:concentrado de 40:60. O acesso dos animais à alimentação foi livre, permitindo $10 \%$ de sobras nos comedouros. A composição bromatológica da dieta experimental foi de $89,5 \%$ de matéria seca; $16,9 \%$ de proteína bruta; $0,69 \%$ de extrato etéreo; e $6,2 \%$ de matéria mineral.

Os cordeiros foram abatidos ao atingirem $25 \mathrm{~kg}$ de peso vivo, em média aos cinco meses de idade. Imediatamente após o abate, nos músculos Biceps femoris, Longissimus e Triceps brachii (lado equerdo da carcaça) foi determinada a cor por meio do colorímetro Minolta CR-200, o qual determina as coordenadas $\mathrm{L}^{*}$, $\mathrm{a}^{*}$ e $\mathrm{b}^{*}$, responsáveis pela luminosidade, teor de vermelho e teor de amarelo, respectivamente (Miltenburg et al., 1992).

Após o período de resfriamento em câmara frigorífica (24 horas), os músculos foram retirados e as carnes submetidas ao processo de maturação, embaladas a vácuo e armazenadas em câmara frigorífica durante sete ou 14 dias, à temperatura de $4^{\circ} \mathrm{C}$. A solução de cloreto de cálcio $0,3 \mathrm{M}$ foi preparada com água destilada e injetada na carne na proporção de $10 \%$ do peso da peça (Jaturasitha et al., 2004). A capacidade de retenção de água foi determinada conforme metodologia descrita por Silva Sobrinho (1999) e a força de cisalhamento de acordo com Osório et al. (1998).

O delineamento experimental utilizado foi em blocos ao acaso, em esquema fatorial $3 \times 2$, com seis tratamentos e quatro blocos, sendo avaliados o tempo de maturação (0, 7 e 14 dias) e a injeção com cloreto de cálcio $(0$ e $0,3 \mathrm{M})$. Os tratamentos consistiram em: controle; maturação durante sete dias; maturação durante 14 dias; cloreto de cálcio 0,3M; maturação durante sete dias e cloreto de cálcio 0,3M; e maturação por 14 dias e cloreto de cálcio $0,3 \mathrm{M}$. As análises de variância foram realizadas de acordo com o SAS (User's..., 1996) e as comparações dos contrastes entre as médias dos tratamentos foram feitas pelo teste Tukey a $5 \%$.

\section{RESULTADOS E DISCUSSÃO}

Na Tab. 1 são apresentadas as medidas de cor da carne. Os músculos apresentaram diferenças na 
cor $\left(L^{*}, a^{*}\right.$ e $\left.b^{*}\right) 24$ horas após o abate. Essas diferenças estão relacionadas com a proporção dos diferentes tipos de fibras nos músculos. Naqueles em que predominam fibras do tipo I, o valor de $\mathrm{a}^{*}$ tende a ser maior - devido ao elevado teor de mioglobina - que naqueles com predomínio de fibras do tipo IIB. Estas apresentam coloração mais clara devido ao menor teor de mioglobina (Prata, 1999).

Para a luminosidade $\left(\mathrm{L}^{*}\right)$, as carnes mais pálidas foram provenientes dos músculos Biceps femoris $(38,94)$ e Triceps brachii, que não diferiram entre si $(\mathrm{P}>0,05)$, e as carnes mais escuras foram oriundas do músculo Longissimus.
O teor de vermelho $\left(\mathrm{a}^{*}\right)$ foi maior no Triceps brachii cujo valor não diferiu do Longissimus, o valor deste foi intermediário e não diferiu do valor do Biceps femoris. Kannan et al. (2001), ao trabalharem com carne de cabras espanholas, relataram valores mais elevados de teor de vermelho em $\mathrm{pH}$ mais elevado. Neste experimento, o músculo Triceps brachii apresentou $\mathrm{pH}$ final mais elevado $(5,93)$ e teor de vermelho maior em relação ao músculo Biceps femoris.

Para o teor de amarelo $\left(\mathrm{b}^{*}\right)$, ocorreu o mesmo, isto é, a ordem dos músculos foi: Biceps femoris, Longissimus e Triceps brachii.

Tabela 1. Cor (L*, a* e b*) proveniente dos músculos Biceps femoris, Longissimus e Triceps brachii em cordeiros da raça Morada Nova após a instalação do rigor mortis

\begin{tabular}{lccc}
\hline Músculo & $\mathrm{L}^{*}$ & $\mathrm{a}^{*}$ & $\mathrm{~b}^{*}$ \\
\hline Biceps femoris & $38,94 \mathrm{a}$ & $15,33 \mathrm{~b}$ & $1,28 \mathrm{~b}$ \\
Longissimus & $37,52 \mathrm{~b}$ & $16,08 \mathrm{ab}$ & $1,77 \mathrm{ab}$ \\
Triceps brachii & $39,74 \mathrm{a}$ & $16,58 \mathrm{a}$ & $2,33 \mathrm{a}$ \\
\hline Teste F & 14,42 & 7,45 & 7,60 \\
DMS & 1,28 & 1,00 & $0,83 \mathrm{~b}$ \\
P & 0,0051 & 0,0237 & 0,0227 \\
CV $(\%)$ & 1,53 & 2,88 & 21,28 \\
\hline
\end{tabular}

Valores seguidos por letras distintas na coluna diferem entre si pelo teste Tukey $(\mathrm{P}<0,05)$. DMS: diferença mínima significativa; P: probabilidade; CV: coeficiente de variação.

Em ovinos são citadas variações de 30,03 a 49,47 para $L^{*}$, de 8,24 a 23,53 para a* e de 3,38 a 11,10 para $b^{*}$ (Sañudo et al., 2000; Warris, 2003).

$\mathrm{Na}$ Tab. 2 são apresentadas as medidas de cor da carne do músculo Biceps femoris em cordeiros da raça Morada Nova. O tempo de maturação influenciou $(\mathrm{P}<0,05)$ a luminosidade. Essa característica, entretanto, não foi influenciada pelo fator cloreto de cálcio $(\mathrm{P}>0,05)$. As carnes maturadas durante 14 dias apresentaram-se mais escuras, pois o valor de $\mathrm{L}^{*}$ foi menor em relação às carnes não maturadas.

Peixoto et al. (2002), ao estudarem as características da carne bubalina maturada, concluíram que o tempo de maturação influiu negativamente na cor dos cortes da carne. $\mathrm{Na}$ avaliação subjetiva da cor, a carne maturada obteve a preferência dos julgadores no início do processo. Ao final, no entanto, a aceitação maior foi para as carnes não maturadas.
O tempo de maturação e o cloreto de cálcio afetaram $(\mathrm{P}<0,05)$ a característica teor de vermelho. As carnes maturadas durante 14 dias e injetadas com cloreto de cálcio $0,3 \mathrm{M}$ apresentaram-se mais escuras em relação às não maturadas e às não injetadas com cloreto de cálcio $0,3 \mathrm{M}$.

De acordo com Arima et al. (1997), citados por Peixoto et. al. (2002), a carne maturada, mesmo após a equalização da cor, ainda apresenta um gradiente diferenciado quando comparada à carne não maturada, pois o ferro presente na mioglobina em baixas pressões de oxigênio passa para a forma oxidada $\left(\mathrm{Fe}^{+++}\right)$, originando a metamioglobina, que apresenta coloração escura (Roça, 2000).

Para os valores de $\mathrm{b}^{*}$, não houve influência $(\mathrm{P}>0,05)$ do tempo de maturação e do cloreto de cálcio para o músculo estudado. Entretanto, de acordo com Sañudo (2002), o aumento no tempo de maturação da carne tende a torná-la mais escura e mais marron, ou seja, o teor de b* tende a ser maior. 
Tabela 2. Cor ( $\mathrm{L}^{*}, \mathrm{a}^{*}$ e $\left.\mathrm{b}^{*}\right)$ da carne proveniente do músculo Biceps femoris em cordeiros da raça Morada Nova nos diferentes tempos de maturação e nas diferentes concentrações de cloreto de cálcio

\begin{tabular}{lccc}
\hline Tempo de maturação $(\mathrm{M})$ & $\mathrm{L}^{*}$ & $\mathrm{a}^{*}$ & $\mathrm{~b}^{*}$ \\
\hline Não maturada & $42,73 \mathrm{a}$ & $14,33 \mathrm{~b}$ & 3,56 \\
7 dias de maturação & $41,80 \mathrm{ab}$ & $15,11 \mathrm{ab}$ & 2,86 \\
14 dias de maturação & $40,03 \mathrm{~b}$ & $16,25 \mathrm{a}$ & 3,66 \\
Teste F & 3,80 & 4,07 & 0,66 \\
$\mathrm{P}$ & 0,05 & 0,04 & 0,53 \\
DMS & 2,58 & 1,76 & 1,97 \\
\hline Concentração de cloreto de cálcio $(\mathrm{C})$ & & & 2,95 \\
\hline Sem cloreto de cálcio & 41,92 & $15,56 \mathrm{~b}$ & 3,76 \\
Com cloreto de cálcio 0,3M & 41,12 & $15,90 \mathrm{a}$ & 1,70 \\
Teste F & 0,98 & 5,94 & 0,21 \\
P & 0,34 & 0,03 & 1,32 \\
DMS & 1,73 & 1,18 & 0,86 \\
\hline Interação MxC & & & 0,44 \\
Teste F & 0,14 & 2,30 & 45,24 \\
P & 0,87 & 0,14 & 8,88 \\
CV(\%) & 4,78 & D. & \\
\hline
\end{tabular}

Dentro de um mesmo fator, médias seguidas por letras distintas na coluna, diferem entre si pelo teste Tukey. DMS: diferença mínima significativa; P: probabilidade; CV: coeficiente de variação.

Também, não houve efeito da interação tempo de maturação versus concentração de cloreto de cálcio para todas as características avaliadas.

Na Tab. 3 são apresentadas as medidas de cor da carne do músculo Longissimus em cordeiros da raça Morada Nova.
Os fatores tempo de maturação e injeção com cloreto de cálcio não influenciaram $(\mathrm{P}>0,05)$ a luminosidade e o teor de amarelo $\left(b^{*}\right)$ do músculo Longissimus. Zapata et al. (2005) também não observaram efeito do tempo de maturação sobre o teor de $\mathrm{L}^{*}$ da carne caprina proveniente dos músculos Biceps femoris e Semimembranosus.

Tabela 3. Cor ( $\mathrm{L}^{*}, \mathrm{a}^{*}$ e $\left.\mathrm{b}^{*}\right)$ da carne proveniente do músculo Longissimus em cordeiros da raça Morada Nova nos diferentes tempos de maturação e nas diferentes concentrações de cloreto de cálcio

\begin{tabular}{lccc}
\hline Tempo de maturação $(\mathrm{M})$ & $\mathrm{L}^{*}$ & $\mathrm{a}^{*}$ & $\mathrm{~b}^{*}$ \\
\hline Não maturada & 41,64 & $14,95 \mathrm{~b}$ & 4,64 \\
7 dias de maturação & 40,39 & $16,17 \mathrm{ab}$ & 3,98 \\
14 dias de maturação & 40,17 & $16,93 \mathrm{a}$ & 4,58 \\
Teste F & 1,13 & 6,67 & 0,65 \\
$\mathrm{P}$ & 0,35 & 0,01 & 0,54 \\
DMS & 2,74 & 1,42 & 1,66 \\
\hline Concentração de cloreto de cálcio (C) & & & 3,91 \\
\hline Sem cloreto de cálcio & 40,51 & 15,83 & 4,89 \\
Com cloreto de cálcio 0,3M & 40,96 & 16,21 & 3,52 \\
Teste F & 0,27 & 0,71 & 0,08 \\
P & 0,61 & 0,44 & 1,11 \\
DMS & 1,83 & 0,95 & 4,34 \\
\hline Interação MxC & & & $0,03^{*}$ \\
Teste F & 0,58 & 0,54 & 28,97 \\
P & 0,57 & 0,59 & \\
\hline
\end{tabular}

Dentro de um mesmo fator, médias seguidas por letras distintas na coluna diferem entre si pelo teste Tukey. DMS: diferença mínima significativa; P: probabilidade; $\mathrm{CV}$ : coeficiente de variação. 
Todavia, para o teor de vermelho, houve efeito $(\mathrm{P}<0,05)$ do tempo de maturação, pois se observaram carnes mais vermelhas quando o tempo de maturação foi de 14 dias em relação às não maturadas. A maturação por sete dias comportou-se de maneira intermediária. Para o fator cloreto de cálcio não houve efeito sobre L*, $a^{*}$ e b*.

Ao avaliarem a cor da carne não maturada do músculo Longissimus dorsi de cordeiros, Souza et al. (2004) observaram teor de a* $(14,84)$ próximo ao deste estudo, que foi de 14,95. Prado et al. (2005) não observaram diferença $(P>0,05)$ na cor da carne proveniente do músculo Longissimus dorsi de bovinos submetida a diferentes períodos de maturação, 7, 14, 30 e 60 dias, cujos valores foram: 39,$73 ; 22,63$ e 14,08 para $L^{*}, a^{*}$ e $b^{*}$, respectivamente.

A interação tempo de maturação versus concentração de cálcio não foi significativa para $\mathrm{L}^{*}$ e $\mathrm{a}^{*}$, mostrando que para essas características os fatores agem independentemente. Quanto ao teor de $b^{*}$, a interação foi significativa, fato que demonstra que a ação do tempo de maturação e da concentração de cloreto de cálcio são interdependentes. $\mathrm{Na}$ Tab. 4 mostram-se os resultados do desdobramento dessa interação. Houve efeito da maturação dentro das concentrações de cloreto de cálcio. As carnes maturadas por sete dias que não receberam cloreto de cálcio apresentaram menor teor de b*, 2,59, enquanto que as que receberam apresentaram maior teor de $b^{*}, 5,38$.

Tabela 4. Desdobramento da interação tempo de maturação x concentração de cloreto de cálcio, para o parâmetro b* (teor de amarelo) da carne proveniente do músculo Longissimus em cordeiros da raça Morada Nova

\begin{tabular}{lccccc}
\hline Tratamento & $\begin{array}{c}\text { Sem } \\
\text { maturação }\end{array}$ & $\begin{array}{c}\text { 7 dias de } \\
\text { maturação }\end{array}$ & $\begin{array}{c}\text { 14 dias de } \\
\text { maturação }\end{array}$ & Teste F & P \\
\hline Sem $\mathrm{CaCl}_{2}$ & $5,12 \mathrm{Aa}$ & $2,59 \mathrm{Ab}$ & $4,03 \mathrm{Aa}$ & 3,98 & 0,04 \\
Com $0,3 \mathrm{M} \mathrm{CaCl}_{2}$ & $4,16 \mathrm{Aa}$ & $5,38 \mathrm{Aa}$ & $5,13 \mathrm{Aa}$ & 1,01 & 0,39 \\
\hline Teste $\mathrm{F}$ & 1,13 & 9,58 & 1,49 & & \\
$\mathrm{P}$ & 0,30 & 0,01 & 0,24 & & \\
\hline
\end{tabular}

$\mathrm{CaCl}_{2}$ : cloreto de cálcio. Dentro de um mesmo fator, médias seguidas por letras minúsculas distintas na coluna diferem entre si pelo teste Tukey. Dentro de um mesmo fator, médias seguidas por letras maiúsculas iguais na linha não diferem entre si pelo teste Tukey. P: probabilidade.

$\mathrm{Na}$ Tab. 5 são apresentadas as medidas de cor da carne do músculo Triceps brachii em cordeiros da raça Morada Nova.

As características $\mathrm{L}^{*} \mathrm{e} \mathrm{a}^{*}$ foram influenciadas $(\mathrm{P}<0,05)$ pelo tempo de maturação. Em relação à luminosidade, as carnes não maturadas apresentaram-se mais pálidas em relação às maturadas durante 14 dias e estas não diferiram $(\mathrm{P}>0,05)$ das maturadas durante sete dias $(41,29)$.

Para o teor de $\mathrm{a}^{*}$, as carnes submetidas ao processo de maturação durante sete e 14 dias não diferiram entre si $(\mathrm{P}>0,01)$, com média de 17,15 , mas apresentaram-se $(\mathrm{P}<0,01)$ mais vermelhas que as carnes não maturadas. A variável $\mathrm{a}^{*}$ indica o teor de vermelho e está relacionada com o pigmento do músculo, a mioglobina, quanto maior for seu valor, mais vermelha será a carne.

Zapata et al. (2005), ao estudarem a cor da carne caprina proveniente dos músculos Biceps femoris e Semimembranosus, relataram que durante o período de maturação a carne caprina apresentou tendência de escurecimento da coloração vermelha, com valores de a* de 16,19 para as carnes não maturadas e 17,12 para as carnes maturadas por sete dias. No presente experimento, o teor de vermelho tendeu a ser maior nas carnes maturadas por 14 dias provenientes dos músculos avaliados.

O tempo de maturação não afetou $(\mathrm{P}>0,05)$ o teor de $\mathrm{b}^{*}$ da carne proveniente do Triceps brachii, com média de 3,33. Zapata et al. (2005) também não observaram efeito do tempo de maturação no teor de amarelo de carne caprina proveniente dos músculos Biceps femoris e Semimembranosus, com valor de b* de $-0,42$ para carnes não maturadas e 0,59 para carnes maturadas por sete dias. 
Tabela 5. Cor $\left(\mathrm{L}^{*}, \mathrm{a}^{*}\right.$ e $\left.\mathrm{b}^{*}\right)$ da carne proveniente do músculo Triceps brachii em cordeiros da raça Morada Nova nos diferentes tempos de maturação e nas diferentes concentrações de cloreto de cálcio

\begin{tabular}{lccc}
\hline Tempo de maturação $(\mathrm{M})$ & $\mathrm{L}^{*}$ & $\mathrm{a}^{*}$ & $\mathrm{~b}^{*}$ \\
\hline Não maturada & $43,15 \mathrm{a}$ & $15,14 \mathrm{~b}$ & 3,35 \\
7 dias de maturação & $41,29 \mathrm{ab}$ & $16,69 \mathrm{a}$ & 2,73 \\
14 dias de maturação & $40,16 \mathrm{~b}$ & $17,61 \mathrm{a}$ & 3,91 \\
Teste F & 5,07 & 17,79 & 1,17 \\
$\mathrm{P}$ & 0,02 & 0,0001 & 0,34 \\
DMS & 2,46 & 1,09 & 2,00 \\
\hline Concentração de cloreto de cálcio (C) & & & \\
\hline Sem cloreto de cálcio & 41,08 & 16,22 & 2,77 \\
Com cloreto de cálcio 0,3M & 41,98 & 16,74 & 3,89 \\
Teste F & 1,36 & 2,36 & 3,17 \\
P & 0,261 & 0,14 & 0,09 \\
DMS & 1,65 & 0,73 & 1,34 \\
\hline Interação MxC & & & \\
\hline Teste F & 0,39 & 5,14 & 0,04 \\
P & 0,68 & 0,02 & 0,96 \\
CV $(\%)$ & 4,56 & 5,08 & 46,31 \\
\hline
\end{tabular}

Dentro de um mesmo fator, médias seguidas por letras distintas na coluna, diferem entre si pelo teste Tukey. DMS: diferença mínima significativa; P: probabilidade; CV: coeficiente de variação.

$\mathrm{O}$ fator cloreto de cálcio não afetou $(\mathrm{P}>0,05)$ os parâmetros $L^{*}, a^{*}$ e $b^{*}$ das carnes. O efeito da interação não foi significativo $(\mathrm{P}>0,05)$ para as características $L^{*} \mathrm{e}^{*}$, mas o foi para a característica $a^{*}(\mathrm{P}<0,05)$.

$\mathrm{Na}$ Tab. 6 apresentam-se os resultados do desdobramento dessa interação. Houve efeito da maturação dentro das concentrações de cloreto de cálcio. As carnes que não receberam cloreto de cálcio apresentaram menor teor de $\mathrm{a}^{*}, 15,66$, enquanto que as que o receberam apresentaram maior valor, 17,73 .

Também houve efeito da concentração de cloreto de cálcio dentro dos tempos de maturação. Nas carnes sem cloreto de cálcio, o teor de vermelho foi maior para as maturadas durante 14 dias, 17,79. Nas carnes com $0,3 \mathrm{M}$ de cloreto de cálcio, o teor de vermelho foi menor, 15,08, para as não maturadas.

Tabela 6. Desdobramento da interação tempo de maturação x concentração de cloreto de cálcio, para o parâmetro a* (teor de vermelho) da carne proveniente do músculo Triceps brachii em cordeiros da raça Morada Nova

\begin{tabular}{lccccc}
\hline Tratamento & $\begin{array}{c}\text { Sem } \\
\text { maturação }\end{array}$ & $\begin{array}{c}7 \text { dias de } \\
\text { maturação }\end{array}$ & $\begin{array}{c}14 \text { dias de } \\
\text { maturação }\end{array}$ & Teste $\mathrm{F}$ & $\mathrm{P}$ \\
\hline $\mathrm{Sem} \mathrm{CaCl}_{2}$ & $15,20 \mathrm{Ba}$ & $15,66 \mathrm{Bb}$ & $17,79 \mathrm{Aa}$ & 10,92 & 0,001 \\
$\mathrm{Com} 0,3 \mathrm{M} \mathrm{CaCl}_{2}$ & $15,08 \mathrm{Ba}$ & $17,73 \mathrm{Aa}$ & $17,42 \mathrm{Aa}$ & 12,00 & 0,001 \\
\hline Teste $\mathrm{F}$ & 0,04 & 12,20 & 0,39 & & \\
$\mathrm{P}$ & 0,84 & 0,003 & 0,54 & & \\
\hline
\end{tabular}

$\mathrm{CaCl}_{2}$ : cloreto de cálcio. Dentro de um mesmo fator, médias seguidas por letras minúsculas distintas na coluna diferem entre si pelo teste Tukey. Dentro de um mesmo fator, médias seguidas por letras maiúsculas distintas na linha diferem entre si pelo teste Tukey. P: probabilidade.

$\mathrm{Na}$ Tab. 7 está apresentada a capacidade de retenção de água (expressa em \% de água retida) da carne em cordeiros da raça Morada Nova. O fator tempo de maturação não afetou $(\mathrm{P}>0,05)$ a capacidade de retenção de água dos músculos Biceps femoris e Longissimus, com médias de 57,3 e $56,9 \%$, respectivamente. Contudo, a capacidade de retenção de água no músculo Triceps brachii foi influenciada pelo tempo de maturação; as carnes não maturadas apresentaram maior capacidade de retenção de água que as maturadas durante sete e 14 dias; estas não diferiram entre si, com valor médio de $55,2 \%$. 
De acordo com Roça (2000), durante a maturação da carne ocorre um leve aumento na capacidade de retenção de água, devido à pequena elevação no $\mathrm{pH}$ e à degradação enzimática da estrutura miofibrilar. Entretanto, esse fato não ocorreu nesta pesquisa.
Silva Sobrinho et al. (2005) observaram que as carnes não maturadas apresentaram maior capacidade de retenção de água $(73,10 \%)$ em relação àquelas maturadas durante sete dias, $65,0 \%$ e 14 dias, $62,9 \%$.

Tabela 7. Capacidade de retenção de água (\%) da carne proveniente dos músculos Biceps femoris, Longissimus e Triceps brachii em cordeiros da raça Morada Nova nos diferentes tempos de maturação e nas diferentes concentrações de cloreto de cálcio

\begin{tabular}{lccc}
\hline Tempo de maturação (M) & Biceps femoris & Longissimus & Triceps brachii \\
\hline Não maturada & 60,05 & 59,06 & $66,19^{\mathrm{a}}$ \\
7 dias de maturação & 55,71 & 56,20 & $55,64^{\mathrm{b}}$ \\
14 dias de maturação & 56,08 & 55,52 & $54,68^{\mathrm{b}}$ \\
Teste F & 1,19 & 1,05 & 6,94 \\
P & 0,33 & 0,37 & 0,01 \\
DMS & 8,09 & 6,74 & 8,90 \\
\hline Concentração de cloreto de cálcio (C) & & 56,29 & 59,92 \\
\hline Sem cloreto de cálcio & 57,40 & 57,56 & 57,75 \\
Com cloreto de cálcio 0,3 M & 57,16 & 0,36 & 0,60 \\
Teste F & 0,01 & 0,56 & 0,45 \\
P & 0,92 & 4,52 & 5,97 \\
DMS & 5,42 & & 1,07 \\
\hline Interação MxC & & 0,70 & 0,3668 \\
\hline Teste F & 0,54 & 0,5125 & 11,65 \\
P & 0,5939 & 9,12 & \\
CV(\%) & 10,86 & & \\
\hline
\end{tabular}

Dentro de um mesmo fator, médias seguidas por letras distintas na coluna diferem entre si pelo teste Tukey. DMS: diferença mínima significativa; P: probabilidade; CV: coeficiente de variação.

O fator cloreto de cálcio não afetou a capacidade de retenção de água dos músculos Biceps femoris, Longissimus e Triceps brachii, com valores médios de 57,3; 56,9 e 58,8\%, respectivamente. Zeola et al. (2005) observaram diferença $(\mathrm{P}<0,05)$ na capacidade de retenção de água da carne de ovelhas proveniente do músculo Longissimus. As carnes que não receberam cloreto de cálcio apresentaram menor capacidade de retenção de água $(53,1 \%)$ em relação à do tratamento-controle $(57,2 \%)$. Do mesmo modo, Pérez et al. (1998) em trabalho de avaliação do efeito da injeção com cloreto de cálcio sobre a capacidade de retenção de água do músculo Biceps femoris de bovinos, observaram que essa característica foi menor para as carnes tratadas com cloreto de cálcio em comparação às amostras-controle, provavelmente devido à proteólise que ocorreu quando a carne recebeu cloreto de cálcio. Não houve efeito da interação dos fatores avaliados $(\mathrm{P}>0,05)$.
$\mathrm{Na}$ Tab. 8 está apresentada a maciez da carne, medida por meio da força de cisalhamento em cordeiros da raça Morada Nova. O tempo de maturação não influenciou $(\mathrm{P}>0,05)$ a maciez do músculo Triceps brachii, com valor médio de $1,70 \mathrm{kgf} / \mathrm{cm}^{2}$, entretanto, influenciou $(\mathrm{P}<0,05)$ a maciez dos músculos Biceps femoris e Longissimus.

No músculo Biceps femoris a maciez foi maior nas carnes maturadas durante sete e 14 dias, e estas não diferiram da maciez com sete dias de maturação $(\mathrm{P}>0,05)$. Para 0 músculo Longissimus, o processo foi aproximadamente o mesmo, isto é, observaram-se melhores valores de maciez para carnes maturadas durante sete e 14 dias $\left(1,89 \mathrm{kgf} / \mathrm{cm}^{2}\right)$, mas estas não diferiram entre si $(\mathrm{P}>0,05)$. 
Tabela 8. Força de cisalhamento (FC) da carne proveniente dos músculos Biceps femoris, Longissimus e Triceps brachii em cordeiros Morada Nova nos diferentes tempos de maturação e nas diferentes concentrações de cloreto de cálcio

\begin{tabular}{lccc}
\multirow{2}{*}{ Tempo de maturação $(\mathrm{M})$} & \multicolumn{3}{c}{$\mathrm{FC}\left(\mathrm{kgf} / \mathrm{cm}^{2}\right)$} \\
\cline { 2 - 4 } \multicolumn{1}{c}{ Não maturada } & Biceps femoris & Longissimus & Triceps brachii \\
7 dias de maturação & $2,76 \mathrm{a}$ & $3,03 \mathrm{a}$ & 1,96 \\
14 dias de maturação & $2,20 \mathrm{ab}$ & $2,21 \mathrm{~b}$ & 1,85 \\
\hline Teste F & $1,76 \mathrm{~b}$ & $1,89 \mathrm{~b}$ & 1,30 \\
P & 6,00 & 4,16 & 3,60 \\
DMS & 0,01 & 0,04 & 0,05 \\
\hline Concentração de cloreto de cálcio (C) & 0,75 & 1,06 & 0,6811 \\
\hline Sem cloreto de cálcio & & & \\
Com cloreto de cálcio 0,3M & 2,29 & $2,89 \mathrm{a}$ & 1,91 \\
\hline Teste F & 2,19 & $1,86 \mathrm{~b}$ & 1,50 \\
P & 0,20 & 9,61 & 3,77 \\
DMS & 0,66 & 0,01 & 0,07 \\
\hline Interação MxC & 0,50 & 0,71 & 0,46 \\
\hline Teste F & & & \\
P & 1,80 & 0,10 & 0,01 \\
CV(\%) & 0,20 & 0,90 & 0,99 \\
\hline D & 25,88 & 34,30 & 30,80 \\
\hline
\end{tabular}

Dentro de um mesmo fator, médias seguidas por letras distintas na coluna diferem entre si pelo teste Tukey. DMS: diferença mínima significativa; P: probabilidade; CV: coeficiente de variação.

Zapata et al. (2003), ao avaliarem a maciez no músculo Longissimus dorsi de cordeiros, também observaram que as amostras não maturadas apresentavam maior força de cisalhamento $(8,86 \mathrm{kgf})$ em comparação com as amostras maturadas durante 21 dias $(6,77 \mathrm{kgf})$.

A injeção com cloreto de cálcio não influenciou $(\mathrm{P}>0,05)$ a maciez dos músculos Biceps femoris $\mathrm{e}$ Triceps brachii, com valores médios de 2,24 e $1,71 \mathrm{kgf} / \mathrm{cm}^{2}$, respectivamente. Entretanto, a maciez do músculo Longissimus foi afetada $(\mathrm{P}<0,05)$ pela injeção com cloreto de cálcio. As carnes injetadas com $0,3 \mathrm{M}$ de cloreto de cálcio foram mais macias quando comparadas com as não injetadas. Zeola et al. (2005) também observaram diferença $(\mathrm{P}<0,05)$ na força de cisalhamento da carne de ovelhas proveniente do músculo Longissimus, quando submetida ao tratamento-controle e ao tratamento com $0,3 \mathrm{M}$ de cloreto de cálcio, com valores de $4,00 \mathrm{kgf} / \mathrm{cm}^{2}$ e $2,95 \mathrm{kgf} / \mathrm{cm}^{2}$, respectivamente. Não houve efeito da interação dos fatores estudados.

\section{CONCLUSÕES}

Os resultados demonstraram que a carne ovina avaliada está contida nos parâmetros qualitativos considerados adequados.

\section{REFERÊNCIAS BIBLIOGRÁFICAS}

ARIMA, H.K.; GARCIA, A.O.; YAMADA, E.A. et al. Buffalo meta ageing at 0 to $2^{\circ} \mathrm{C}$. In: WORLD BUFFALO CONGRESS, 5., 1997, Caserta. Anais... Caserta, 1997.

JATURASITHA, S.; THIRAWONG, P.; LEANGWUNTA, V. et al. Reducing toughness of beef from Bos indicus draught steers by injection of calcium chloride. Effect of concentration and time post mortem. Meat Sci., v.68, p.61-69, 2004.

KANNAN, G.; CHAWAN, C.B.; KOUAKOU, B. et al. Color changes reflecting myoglobin and lipid oxidation in chevon cuts during refrigerated display. Small Rum. Res., v.42, p.67-75, 2001.

MARTÍNEZ-CEREZO, S.; SAÑUDO, C.; PANEA, B. et al. Breed, slaughter weight and ageing time effects on consumer appraisal of three muscles of lamb. Meat Sci., v.69, p.795-805, 2005.

MILTENBURG, G.A.J.; WENSING, T.H.; SMULDERS, F.J.M. et al. Relationship between blood hemoglobin, plasma and tissue iron, muscle heme pigment, and carcass color of veal. J. Anim. Sci., v.70, p.2766-2772, 1992.

NUTRIENT requirements of sheep. New York: National Academy, 1985. 99p.

OSÓRIO, J.C.S.; OSÓRIO, M.T.M.; JARDIM, P.O.C. et al. Métodos para avaliação da produção 
de carne ovina: "in vivo", na carcaça e na carne. Pelotas:UFPEL, 1998. 107p.

OSÓRIO, J.C.S.; OSÓRIO, M.T.M. Condições de abate e qualidade de carne. In: CURSO DE QUALIDADE DA CARNE E DOS PRODUTOS CÁRNEOS, 1999, Bagé. Anais... Bagé, 1999. 65p.

PARDI, M.C.; SANTOS, I.F.; SOUZA, E.R. et al. Ciência, higiene e tecnologia da carne. 2.ed. Goiânia: UFG, 2001. 623p.

PEIXOTO, M.R.S.; SOUSA, C.L.; NEVES, E.C.A. Avaliação físico-química, microbiológica e sensorial da carne bubalina maturada sob diferentes aspectos. Rev. Cien. Agr., n.37, p.43-52, 2002.

PÉREZ, M.L.; ESCALONA, H.; GUERRERO, I. Effect of calcium chloride marination on calpain and quality characteristics of meat from chicken, horse, cattle and rabbit. Meat Sci., v.48, p.125-134, 1998.

PRADO, C.S.; BUENO, C.P.; LAGE, M.E. et al. Resfriamento de carcaças com e sem aspersão de água: influência na cor de bifes de contrafilé (m. Longissimus dorsi) maturados por 7, 14, 30 e 60 dias. In: CONGRESSO BRASILEIRO DE CIÊNCIA E TECNOLOGIA DE CARNES, 3., 2005, São Pedro. Anais...São Pedro, 2005.

PRATA, L.F. Higiene e inspeção de carnes, pescado e derivados. Jaboticabal: FUNEP, 1999. $217 \mathrm{p}$.

ROÇA, R.O. Tecnologia da carne e produtos derivados. Botucatu: Faculdade de Ciências Agronômicas, UNESP, 2000. 202p.

SAÑUDO, C. La calidad organoléptica de la carne con especial referencia a la especie ovina. Factores que la determinan, metodos de medida y causas de variacion. 1992. 117f. Facultad de Veterinaria Departamento Producción Animal y Ciencia de los Alimentos, Zaragoza.

SAÑUDO, C.; ENSER, M.E.; CAMPO, M.M. et al. Fatty acid composition and sensory characteristics of lamb carcasses from Britain and Spain. Meat Sci., v.54, p.339-346, 2000.

SAÑUDO, C. Factors affecting carcass and meat quality in lambs. In: REUNIÃO ANUAL DA SOCIEDADE BRASILEIRA DE ZOOTECNIA,
39., 2002, Recife. Anais...Recife, SBZ, 2002. p.434-455.

SAÑUDO, C. Analisis sensorial - Calidad organoléptica de la carne. In: CURSO INTERNACIONAL DE ANALISE SENSORIAL DE CARNE E PRODUTOS CÁRNEOS, 2004, Pelotas. Anais...Pelotas, 2004. p.45-68.

SILVA SOBRINHO, A.G. Body composition and characteristics of carcass from lambs of different genotypes and ages at slaughter, 1999. 54f. Report (PostDoctorate in Sheep Meat Production) - Massey University, Palmerston North, New Zealand.

SILVA SOBRINHO, A.G. Criação de ovinos. Jaboticabal: FUNEP, 2001. 302p.

SILVA SOBRINHO, A.G.; PURCHAS, R.W.; KADIM, I.T. et al. Características de qualidade da carne de ovinos de diferentes genótipos e idades ao abate. Rev. Bras. Zootec., v.34, p.1070-1078, 2005.

SOUZA, X.R.; BRESSAN, M.C.; PÉREZ, J.R.O. et al. Efeitos do grupo genético, sexo e peso ao abate sobre as propriedades físico-químicas da carne de cordeiros em crescimento. Cien. Tecnol. Alim., v.24, p.543-549, 2004.

USER'S guide: statistic. Version 6.12. 4.ed. Cary: SAS Institute, 1996.

WARRIS, P.D. Ciencia de la carne. Zaragoza:Acribia, 2003. 309p.

ZAPATA, J.F.F.; ALMEIDA, R.; SOUZA, D. et al. Physical and functional characteristics of tropical lamb aged for 21 days. In: INTERNATIONAL CONGRESS OF MEAT SCIENCE AND TECHNOLOGY, 49., 2003, Campinas. Anais... Campinas, 2003. p.195-196.

ZAPATA, J.F.F.; PEREIRA, A.L.F.; VIDAL, T.F. et al. Influência do tipo de músculo e da maturação sobre as propriedades funcionais e de maciez da carne caprina. In: CONGRESSO BRASILEIRO DE CIÊNCIA E TECNOLOGIA DE CARNES, 3., 2005, São Pedro. Anais...São Pedro, 2005.

ZEOLA, N.M.B.L.; SILVA SOBRINHO, A.G.; SOUZA, P.A. et al. Avaliação da injeção de cloreto de cálcio nos parâmetros qualitativos da carne de ovelha. Rev. Bras. Agroc., v.11, p.361-364, 2005. 